\title{
Nanofluid Based on Carbon Dots Functionalized with Ionic Liquids for Energy Applications
}

\author{
Helena M. R. Gonçalves ${ }^{1,2, *}$, , Susana A. F. Neves ${ }^{2}$, Abel Duarte ${ }^{2}{ }^{\mathbb{D}}$ and \\ Verónica de Zea Bermudez ${ }^{1, * \text { (D) }}$ \\ 1 Chemistry Department and CQ-VR, University of Trás-os-Montes e Alto Douro, 5001-801 Vila Real, Portugal \\ 2 REQUIMTE, Instituto Superior de Engenharia do Porto, 4200-072 Porto, Portugal; \\ susananeves13@gmail.com (S.A.F.N.); ajd@isep.ipp.pt (A.D.) \\ * Correspondence: helenardrgs@gmail.com (H.M.R.G.); vbermude@utad.pt (V.d.Z.B.)
}

Received: 11 December 2019; Accepted: 29 January 2020; Published: 3 February 2020

check for updates

\begin{abstract}
The development of materials that can help overcome the current limitations in energy storage and consumption is a pressing need. Recently, we developed non-Newtonian nanofluids based on non-toxic, carbon nanoparticles (NPs), carbon dots (Cdots) functionalized with ionic liquids. Here, we wanted to prove that these new nanofluids are, not only interesting as possible electrolytes, but also as new organic/inorganic hybrid separators. As such, we developed an entrapment method using poly(vinyl alcohol) (PVA). Indeed, the highly conductive Cdots were successfully retained inside the membrane even upon the application of several wetting/drying cycles. Moreover, the morphological characteristics did not change upon wetting/drying cycles and remained constant for more than four months. These nanofluids could be an interesting approach to tackle some of the current problems in the fields of solid-state batteries, and energy storage, among others.
\end{abstract}

Keywords: nanofluids; carbon dots; ionic liquids; electrolytes; energy applications

\section{Introduction}

The roadmap for moving into a competitive low carbon economy by 2050 [1] has raised social awareness of the need to develop new alternative technologies that effectively contribute to a significant decrease of greenhouse gas emissions. Battery technologies are at the center of this problem. Indeed, the production of advanced batteries and battery materials that will enable large-scale electrification transport, and the storage of intermittently produced renewable energies, such as wind and solar, are of vital importance.

Lithium $(\mathrm{Li})$ ion batteries have been at the heart of the ongoing portable technology revolution, as recognized recently by the Nobel Prize in Chemistry 2019 [2]. However, the increasing demand for ultrahigh performance (higher storage capacity and power), easy recharging, reliability, safety, and low price cannot be fully satisfied with the existing electrochemistry knowledge and technology. Although there have been major efforts in the development of efficient upgrades [3,4], it can be stated that globally, current research on batteries has two main directions [5]: (1) Advanced Li-ion, which consists of the optimization of the already fully developed and mature Li-ion technology; and (2) post Li-ion, where modifications of its core components are attempted. Regardless of the research direction, the development of new fast-ion conductors or solid electrolytes is at the heart of the solid-state battery concept $[5,6]$. Accelerating research on the design of battery materials and interfaces, promoting research to incorporate smart sensing and self-healing functionalities into future batteries, and guaranteeing manufacturability, scalability, recyclability, and sustainability, are of paramount importance [7]. 
The application of nanofluids in the energy area has been growing exponentially over the past few years, leading to quite interesting developments recently [8,9]. According to Choi et al. [10], nanofluids are stable dispersions of NPs in a base fluid. The introduction of the NPs in the base fluid results in a dramatic increase in the heat transfer performance [11]. This relevant feature of nanofluids has been the boosting factor beneath the current research in this area. The use of nanofluids as efficient heat transfer agents is quite appealing for several industrial areas, particularly in energy applications. Nowadays, the conventional coolants/fluids used in car engines and radiators, for example, contain millimeter/micrometer-sized particles to increase the heat transfer properties. However, they are not very efficient. Moreover, the use of $\mathrm{mm} / \mu \mathrm{m}$-sized particles hampers the use of new cooling technologies based on microchannels, since they easily clog [12]. In this sense, the use of nanofluids with higher thermal conductivity and stability can lead to an enhancement of the engines' and radiators' efficiency, and consequently to the development of lighter vehicles with better fuel economy [13].

Conventional nanofluids contain water, oils and lubricant, and organic liquids, such as, poly(ethylene glycol), bio-fluids, and polymeric solutions, among others [12]. Although ionic liquids (ILs) have been extensively explored in the field of solid-state electrochemistry [14], their use as a base fluid to form nanofluids is scarce, but quite promising $[15,16]$. Indeed, the thermal and electrochemical stability of ILs along with the increase in conductivity when associated with NPs makes them especially appealing to solve fundamental problems, such as the development of rechargeable lithium metal batteries where the uneven deposition of lithium hampers their stability along the repeated charge/discharge cycles [15]. Quite recently, our research group developed unique sustainable nanofluids based on synthetic polysaccharide [17] or waste biomass-derived [18] carbon dots (Cdots) functionalized with imidazolium-based ILs.

Cdots have intrinsic features, such as thermal/electronic conductivity and optoelectronic properties, which are extremely dependent on the surface chemistry. Due to their unique band structure, carbon nanomaterials exhibit a remarkable electron mobility at room temperature. Indeed, electrons in these materials can move with a Fermi velocity $(v F)$ in the ballistic transport regime [19]. Moreover, the electron mobility value in suspended carbon nanomaterials may be as high as about $200,000 \mathrm{~cm} \cdot \mathrm{V}^{-1} \cdot \mathrm{s}^{-1}$, even without considering the charged impurities and ripples [20]. These characteristics make carbon nanomaterials, including Cdots, quite appealing for general energy purposes and in particular for applications where high electronic conductivity is required.

Using the Cdots/IL strategy, we developed three different nanofluids composed of: (1) Glucose-derived Cdots and commercial 1-butyl-3-methylimidazolium chloride ([Bmim]Cl) (Cdots/[Bmim]Cl) [17]; (2) Glucose-derived Cdots, [Bmim]Cl plus in-house-made 1-tosylate-3methyl-imidazolium triflate [Tmi][Trif] (Cdots/[Bmim]Cl/[Tmi][Trif]) [17]; and (3) chitin (from crab shells)-derived Cdots with a mixture of these two ILs (CdotsCHI/[Bmim]Cl/[Tmi][Trif]) [18]. In these systems IL(s) play(s) a dual role: (1) Functionalization molecule and, (2) Reaction medium. Cdots can be produced by several synthetic methods that are often expensive and time consuming. By using ILs it was possible to develop nanofluids using a new hydrothermal, simple, straightforward, and single-step method that requires mild reaction conditions (low reaction times and low temperatures $\left.\left(100{ }^{\circ} \mathrm{C}\right)\right)$. The chosen ILs were fundamental for the synthesis of stable nanofluids, regardless of the carbon source employed for the Cdots production. Indeed, the use of $[\mathrm{Bmim}] \mathrm{Cl}$ enabled the formation of the nanofluid base, and the use of [Tmi][Trif] was responsible for the cleavage of the chitin polymer into its correspondent monomers [21]. Additionally, the use of this sulfonated IL along with [Bmim] Cl allowed an interesting increase in proton conductivity.

Herein, we propose the immobilization in poly(vinyl alcohol) (PVA) of the Cdots/[Bmim]Cl/[Tmi][Trif] and CdotsCHI/[Bmim]Cl/[Tmi][Trif] nanofluids, which exhibit high proton conductivity. The resulting PVA membranes were thoroughly studied in terms of wettability, photostability, and leaching patterns. New results on the fluorescence features of the two nanofluids and [Tmi][Trif] are reported here for the first time. 


\section{Materials and Methods}

\subsection{Materials}

1-Methylimidazole (Merck, 99\%), 1-butyl-3-methylimidazolium chloride ([Bmim]Cl, Acros Organics, 98\%), trifluoromethanesulfonic acid $\left(\mathrm{CF}_{3} \mathrm{SO}_{3} \mathrm{H}\right.$, Merck, $\left.99 \%\right)$, hydrochloric acid $(\mathrm{HCl}$, $37 \%$, Honeywell), nitric acid $\left(\mathrm{HNO}_{3}, 65 \%\right.$, (PanReac), anhydrous acetone (Fluka), poly(vinyl alcohol) (PVA 3-96) Mw 26,300-30,000 g. $\mathrm{mol}^{-1}$, Fluka), glutaraldehyde solution 50\% in $\mathrm{H}_{2} \mathrm{O} 5.6 \mathrm{M}$, (Fluka), chitin (from crab shells, Sigma Aldrich), cyanuric chloride $\left((\mathrm{NCCl})_{3}\right.$, Sigma Aldrich, 99\%), and anhydrous glucose (Fisher Scientific) were used as received unless otherwise stated. High-purity Milli-Q water (resistivity $18 \mathrm{M} \Omega \cdot \mathrm{cm}$, Interlab Sistem Purist UV set) was used in all aqueous solutions.

\subsection{Methods}

\subsubsection{1-Tosylate-3-Methyl-Imidazolium Triflate ([Tmi][Trif])}

The synthesis procedure and the characterization were described elsewhere [18]. Briefly, it occurred in two consecutive steps. In the first step, a solution composed of $\mathrm{CF}_{3} \mathrm{SO}_{3} \mathrm{H}$ in anhydrous acetone in a $(1: 12, v / v)$ ratio was slowly and carefully prepared. In another flask, a solution of 1-methylimidazole in the same solvent and using the molar ratio of the above solution was prepared. The two solutions were mixed under constant stirring and kept at room temperature overnight. The second step consisted in mixing the as-prepared solutions with $10 \mathrm{mmol}$ of $\mathrm{p}$-toluene sulfonic acid under constant stirring in an ice bath. When the temperature of the solution was stable, the mixture was heated to $100{ }^{\circ} \mathrm{C}$ and left under stirring overnight. The resulting [Tmi][Trif] IL was obtained as a black viscous liquid. Upon completion of the reaction, the IL was placed in a vacuum system until no significant change of volume was observed. The purification of [Tmi][Trif] was performed using a dialysis molecular membrane with a molecular mass cutoff of 500-1000 Da. Dialyses was performed against ultrapure water for $48 \mathrm{~h}$. Afterwards, the compound was lyophilized for $72 \mathrm{~h}$, and used without further treatment.

\subsubsection{Nanofluid Synthesis}

The synthesis of the nanofluids was performed according to the procedure reported elsewhere (Figure 1) [17,18]. Cdots obtained from anhydrous glucose (Cdots/[Bmim]Cl/[Tmi][Trif]) and chitin from crab shells (CdotsCHI/[Bmim]Cl/[Tmi][Trif]) were obtained from the addition of [Bmim]Cl to anhydrous glucose or chitin in a $1: 10(\mathrm{~m} / \mathrm{m})$ ratio under constant magnetic stirring, respectively. Afterwards, $1 \mathrm{~mL}$ of pure [Tmi][Trif] was added to the mixtures, and left to react at $100{ }^{\circ} \mathrm{C}$ in an oil bath for $1 \mathrm{~h}$. The resulting NPs were left to cool at room temperature. After cooling, the resulting nanofluids presented a viscous consistency. Cdots/[Bmim]Cl/[Tmi][Trif] had a brown coloration, and $\mathrm{Cdots} \mathrm{CHI} /[\mathrm{Bmim}] \mathrm{Cl} /[\mathrm{Tmi}][$ Trif] exhibited an orange color. Both these materials were transferred into a dialysis cellulose tubing membrane with a molecular weight cutoff of $500 \mathrm{Da}$, and dialyzed against ultrapure water for at least $72 \mathrm{~h}$. The resulting solution was centrifuged for $10 \mathrm{~min}$ at $5000 \mathrm{rpm}$, and the supernatant was extracted and centrifuged again. This procedure was repeated 3 times until no solid could be separated from the solution. The resulting solutions were lyophilized until no water was found (roughly 3 days). The purified nanofluids had a viscous consistency at room temperature. Upon lyophilization, they could easily be re-dispersed in water and other solvents whenever necessary. 


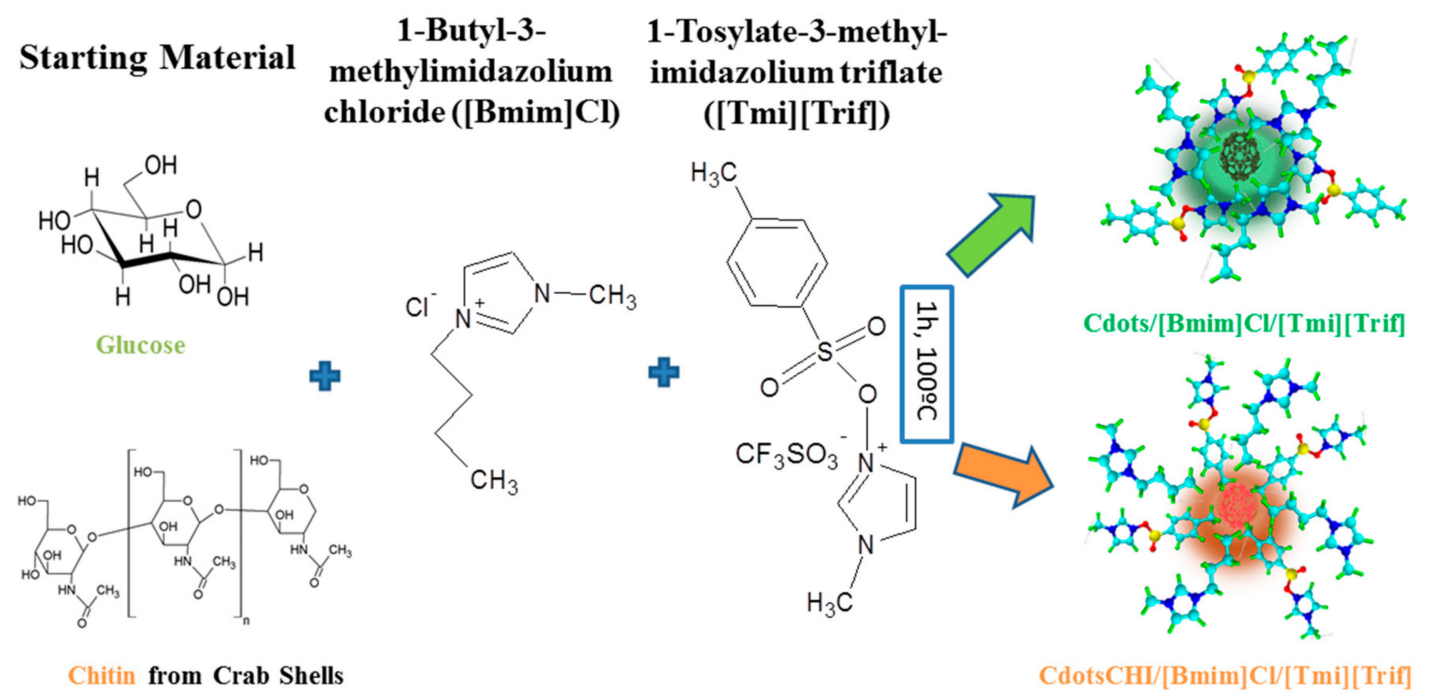

Figure 1. Schematic representation of the single-step synthesis of the Cdots/[Bmim]Cl/[Tmi][Trif] and CdotsCHI/[Bmim]Cl/[Tmi][Trif] nanofluids.

\subsubsection{Preparation of the PVA/Nanofluid Membranes}

The PVA-based membranes were prepared by first dissolving $0.5 \mathrm{~g}$ of $(\mathrm{NCCl})_{3}$ in $20 \mathrm{~mL}$ of acetone. Afterwards, $1.0 \mathrm{~g}$ of PVA and $10 \mathrm{~mL}$ of water were added. The as-prepared mixture was stirred for $30 \mathrm{~min}$ at room temperature. The solid was separated by vacuum filtration, and washed first with acetone and then with water. The PVA/(NCCl $)_{3}$ solid was then covered with a solution containing the nanofluid or [Tmi][Trif] in acetone $(50 \% \mathrm{~m} / \mathrm{v})$. After $1 \mathrm{~h}, 100 \mathrm{mg}$ of the PVA/nanofluid and PVA/[Tmi][Trif] were dissolved in $2.0 \mathrm{~mL}$ of water, in a water bath at $80^{\circ} \mathrm{C}$ for $30 \mathrm{~min}$, until complete dissolution of the PVA. After cooling to room temperature, $0.5 \mathrm{~mL}$ of PVA/nanofluid and PVA/[Tmi][Trif] solution was combined with $0.05 \mathrm{~mL}$ of $2 \%$ aqueous glutaraldehyde (GA) and $0.05 \mathrm{~mL}$ of a $4 \mathrm{M} \mathrm{HCl}$ solution. An aliquot of $50 \mu \mathrm{L}$ was applied on a glass slide previously cleaned with a $\mathrm{HNO}_{3}$ solution $(1: 3 v / v)$ and left to dry at room temperature for $12 \mathrm{~h}$. A thin film was formed in all cases.

\subsection{Characterization}

The conductivity measurements were performed on a WPA CM35 analog conductivity meter with a homemade probe with a constant value of $0.5468 \mathrm{~cm}^{-1}$. Conductivity was acquired as a function of temperature $\left(5\right.$ and $\left.60^{\circ} \mathrm{C}\right)$; as such, during measurements, the sample holder was kept inside a Julabo F25 thermostatic bath.

The dynamic viscosity measurements were performed on a Brookfield DV-II rheometer equipped with a cone-plate system (SSA spindle 21). In each measurement, $2 \mathrm{~mL}$ of the sample was placed inside the small sample adapter that had a double wall to assure thermotatization. The temperature control over the experiments was obtained by using a thermostatic bath Julabo F25. The temperature was changed in a 5 to $60^{\circ} \mathrm{C}$ range. The equipment has an internal calibration provided by the manufacturer, and no further calibration procedures were done.

The stability over the drying and wetting cycles of the PVA membranes was monitored for one month to check any nanofluid leaching from the membrane. The deionized water where the membranes were immersed was tested over time to evaluate any possible residual fluorescence using homemade equipment composed of an electronic circuit to stabilize the electrical current that powered the light source, which constituted a $450 \mathrm{~nm}$ LEDs from Roithner Lasertechnik (Ref. LED450-01), a CCD detector from Ocean Optics (USB2000), and a sampling compartment from Ocean Optics (CUV-ALL-UV 4-way). In all these fluorescence measurements, a $1 \mathrm{~cm}$ quartz cuvette with a magnetic stirring bar was used.

The AFM measurements were performed in tapping mode using an AFM CSI Nano-Observer equipment (Scientec) and a super sharp Si HQ:NSC19/FORTA probe with a frequency resonance of 
$60 \mathrm{kHz}$ and a spring constant of $0.3 \mathrm{~N} \cdot \mathrm{m}^{-1}$. The data collected were analyzed using the Gwyddion 2.52 software. The quality of the images was improved using the flattening and elimination of line noise tools.

The polarized optical microscopy (POM) images were acquired using an OPTIKA B-600 Pol microscope equipped with a digital camera 8 MPx Digital Photo and further analyzed with the Optika Vision Pro software.

\section{Results}

\subsection{Fundamentals}

The high-resolution transmission electron microscopy (HR-TEM) and dynamic light scattering (DLS) data $[17,18]$ proved that the produced Cdots had spherical morphologies and different size distributions depending on the ILs used and the raw carbon material they originated from. The smaller NPs (mean size of $3 \mathrm{~nm}$ ) were found in the Cdots/[Bmim]Cl nanofluid, as expected for NPs obtained with a low water content IL [17]. In this particular case, Cdots were only functionalized with solely one IL. The IL's low interface energy and repulsive effects allowed the NPs' stabilization and prevented their aggregation, which resulted in a well-defined, homogeneous, and small-sized NPs. On the other hand, the Cdots/[Bmim]Cl/[Tmi][Trif] had an average size of $44 \mathrm{~nm}$, whereas CdotsCHI/[Bmim]Cl/[Tmi][Trif] presented two distinct size populations, with average sizes of 44 and $170 \mathrm{~nm}$ [18]. Taking into account the fact that these NPs are functionalized with two ILs with different ionic charge characteristics, it is understandable that these NPs present a larger size when compared to $\mathrm{Cdots} /[\mathrm{Bmim}] \mathrm{Cl}$. Moreover, the higher size heterogeneity observed for CdotsCHI/[Bmim]Cl/[Tmi][Trif], where large micelle-like structures, as well as smaller NPs, were detected, can be due to the production mechanism [22]. Assuming the typical nanoparticle mechanism, it is highly likely that the cleavage of the N-linkages between the chitin carbohydrate monomers assisted by the sulfonated IL [Tmi][Trif] is a slower mechanism that yields larger aggregates than those occurring when glucose is the starting material. Since the nanofluids are sensitive to the surrounding temperature, we performed a DLS analysis before and upon a single heating/cooling cycle, in order to determine if the size distribution was affected by the temperature range used for the conductivity and viscosity measurements $\left(16-50{ }^{\circ} \mathrm{C}\right)$. We observed that in this temperature range, there was no significant change of the nanoparticles' size, as expected, since the size should only change (increase) close to the reaction temperature $\left(100^{\circ} \mathrm{C}\right)$.

Using the information obtained from the DLS analysis, and considering the reagents used for each synthesis, it was possible to calculate the number of IL molecules available for interaction with the Cdots' surface using the method reported by Sun et al. [23]. In this sense, we determined that Cdots/[Bmim]Cl/[Tmi][Trif] had around $2.74 \times 10^{6}$ molecules of $[\mathrm{Bmim}] \mathrm{Cl}$ and $1.96 \times 10^{7}$ molecules of [Tmi][Trif] on the surface. On the other hand, the CdotsCHI/[Bmim]Cl/[Tmi][Trif] nanofluid had two distinct size populations. In this particular, case we calculated the number of IL molecules independently for each population. We determined that the number of [Bmim] $\mathrm{Cl}$ and [Tmi][Trif] molecules available for Cdots' surface interaction was $4.39 \times 10^{6}$ and $4.82 \times 10^{7}$ for the 45 and $170 \mathrm{~nm}$-sized populations, respectively [18].

\section{Nanofluid Characterization}

Cdots are best known for their appealing fluorescence properties, which were fully explored here. Indeed, fluorescent studies can be a powerful tool in this particular case. These nanofluids can be used as common electrolytes or separators (upon immobilization in the PVA membrane). In either case, the possibility of having a property (fluorescence) that allows the electrolyte stability to be deduced, as well as its successful entrapment inside the membrane, is of great interest. Moreover, fluorescence changes with temperature can be followed in real-time real-life situations just by irradiating the sample with a simple black light (ultraviolet (UV)-A light) and a photodetector. In the Cdots case, it is commonly accepted that the fluorescence mechanism is strongly dependent on the raw material and 
the functionalization molecules. Since both surface defects and surface chemical groups are of extreme relevance in the optical properties of the NPs, we analyzed the difference between the fluorescence intensity of the nanofluids obtained from glucose and chitin.

Figure 2a shows that despite the use of the same fluorescence acquisition parameters (excitation wavelength and slits), the emission profile and the fluorescence intensity of both nanofluids are markedly different. Indeed, Cdots/[Bmim] $\mathrm{Cl}$ has a maximum emission wavelength at $495 \mathrm{~nm}$ at a maximum excitation wavelength of $400 \mathrm{~nm}$. Thereby, it has a Stokes shift of $95 \mathrm{~nm}$ and a full width half maximum (FWHM) of $125 \mathrm{~nm}$. On the other hand, the Stokes shift of both Cdots/[Bmim]Cl/[Tmi][Trif] and CdotsCHI/[Bmim]Cl/[Tmi][Trif] is $103 \mathrm{~nm}$ (excitation maximum of $400 \mathrm{~nm}$ and emission maximum of $503 \mathrm{~nm}$ ). It is also interesting to note that the FWHM of the nanofluids functionalized with [Tmi][Trif] are lower than that observed for the nanofluid containing solely [Bmim]Cl. Furthermore, the FWHM of Cdots/[Bmim]Cl/[Tmi][Trif] is $13 \mathrm{~nm}$ higher $(113 \mathrm{~nm})$ than that of the CdotsCHI/[Bmim]Cl/[Tmi][Trif] $(97 \mathrm{~nm}$ ) and $11 \mathrm{~nm}$ superior than that of [Tmi][Trif] (Figure 2b,c).
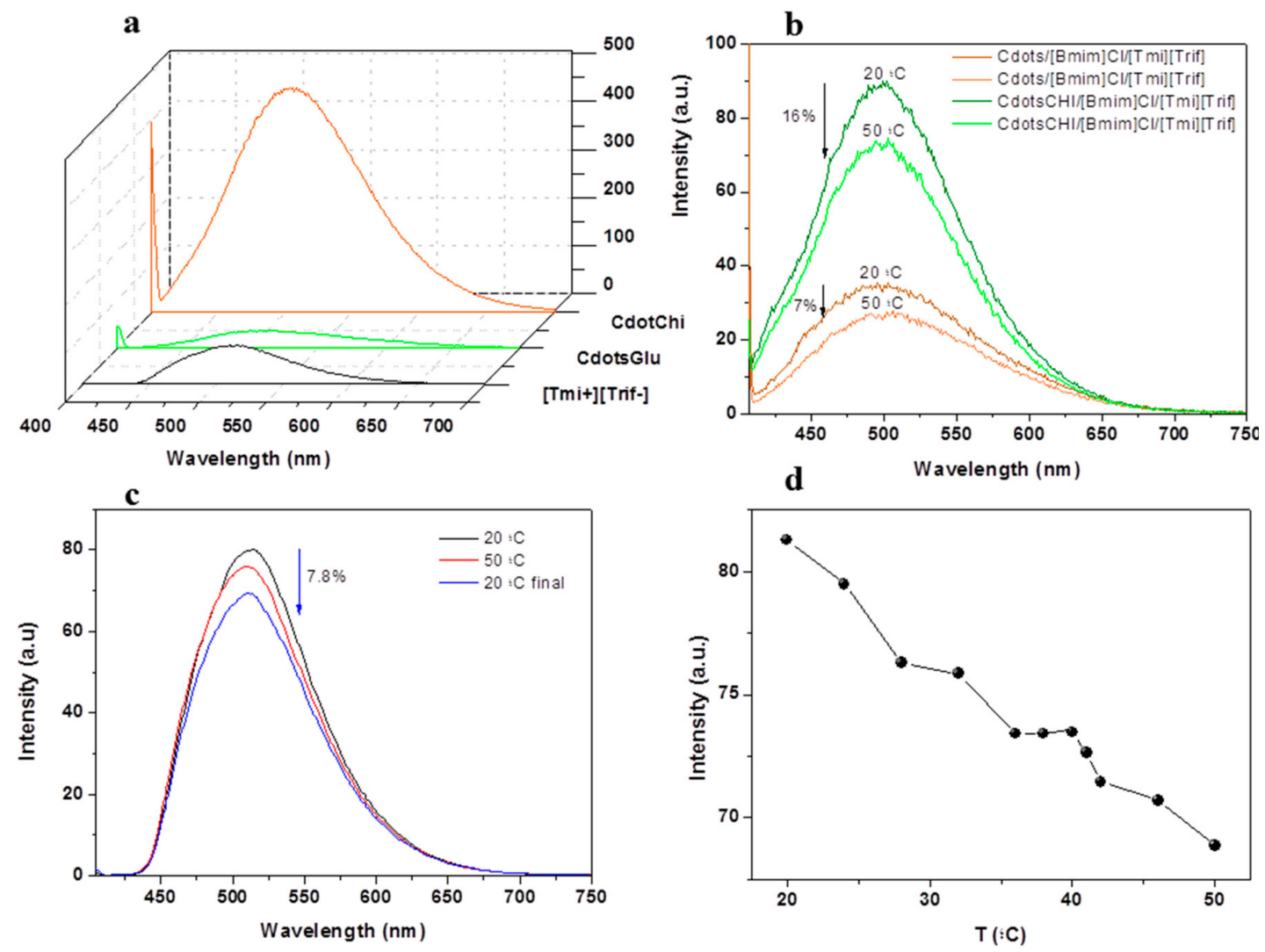

Figure 2. (a) Fluorescence emission profile of the two nanofluids and [Tmi][Trif] (excitation wavelength $=400 \mathrm{~nm}$ ), (b) Fluorescence intensity profile for the two nanofluids aqueous solution acquired at $20^{\circ} \mathrm{C}$, and at the end of the first heating cycle $\left(50^{\circ} \mathrm{C}\right)$. (c) Fluorescence intensity at the maximum emission wavelength of [Tmi][Trif] $(515 \mathrm{~nm})$ as a function of the temperature in the first heating/cooling cycle.

(d) Fluorescence emission profile of [Tmi][Trif] (excitation wavelength $=400 \mathrm{~nm}$ ), at $20{ }^{\circ} \mathrm{C}$, at the maximum temperature of the first heating cycle $\left(50^{\circ} \mathrm{C}\right)$, and at $20^{\circ} \mathrm{C}$ upon the first heating/cooling cycle.

Another interesting feature of the nanofluids and of the [Tmi][Trif] IL is the temperature sensitivity (Figure $2 \mathrm{~b}-\mathrm{d}$ ). Indeed, the fluorescence intensity of both nanofluids decreases and the FWHM increases with increasing temperature, ranging from $4 \mathrm{~nm}$ for Cdots/[Bmim]Cl/[Tmi][Trif] to $3 \mathrm{~nm}$ in Cdots/[Bmim]Cl/[Tmi][Trif] (Figure 2b). In the case of [Tmi][Trif], the temperature sensitivity is also present, but the FWHM only increases $1 \mathrm{~nm}$ in the $20-50{ }^{\circ} \mathrm{C}$ temperature range. This behavior is quite interesting since previous studies attributed a FWHM $>100 \mathrm{~nm}$ to strong electron-electron interaction [24]. In this sense, it is possible to say that at $50{ }^{\circ} \mathrm{C}$, these interactions are superior to those 
observed at $20^{\circ} \mathrm{C}$ in the case of the nanofluids, but less marked for the IL, in agreement with previous studies that explored electron-electron interactions confined in the nanoscale.

Figure $2 \mathrm{~d}$ also shows that upon the first heating/cooling cycle, the fluorescence intensity of [Tmi][Trif] did not reach the initial fluorescence intensity, regardless of the time that it was left at room temperature. This effect is quite relevant and proves that the temperature increase most probably led to the breaking of some hydrogen bonds that were not reformed as the molecules returned to the initial temperature. A $7.8 \%$ decrease in the maximum fluorescence intensity resulted. It is further relevant to note that this effect is masked by the nanofluids. Indeed, and even though they present a similar trend on the heating/cooling cycle, the fluorescence loss upon each cycle does not surpass $1 \%$.

This temperature sensitivity is not restricted to the fluorescence properties. Figure 3a clearly demonstrates that upon the first heating cycle and considering the values obtained for $50{ }^{\circ} \mathrm{C}$, the introduction of [Tmi][Trif] on the Cdots' surface was responsible for the quite significant conductivity increases (from $1.30 \mathrm{mS} \cdot \mathrm{cm}^{-1}$ in Cdots/[Bmim] Cl to $2.79 \mathrm{mS} \cdot \mathrm{cm}^{-1}$ in Cdots/[Bmim]Cl/[Tmi][Trif] and $17.5 \mathrm{mS} \cdot \mathrm{cm}^{-1}$ in $\mathrm{CdotsCHI} /[\mathrm{Bmim}] \mathrm{Cl} /[\mathrm{Tmi}][$ Trif $\left.]\right)(54 \%$ and $92 \%$ increase, respectively). These results are quite appealing, particularly for the field of polymer electrolytes of the next generation of lithium/sodium-ion batteries [25]. The number of works in the literature reporting the use of Cdots for such a purpose is rather scarce. Ma et al. [25] reported a novel electrolyte based on poly(ethylene oxide) (PEO) nanocomposite with oxygen-rich Cdots that exhibited a conductivity of $0.139 \mathrm{mS} \cdot \mathrm{cm}^{-1}$, a value significantly lower than that obtained here using ILs as the surface groups.
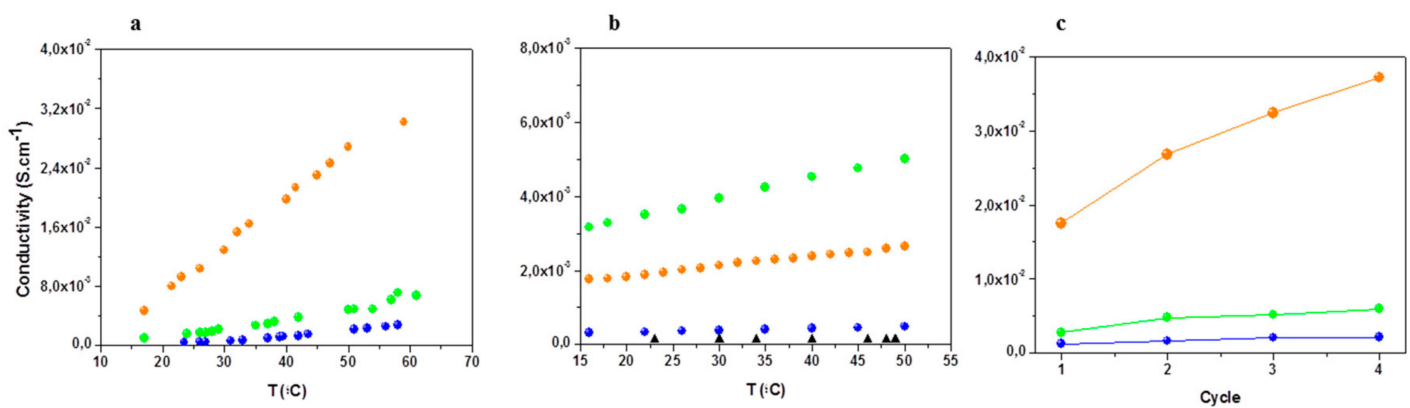

Figure 3. Conductivity profile of the (a) pristine nanofluids; (b) $95 \%(w / w)$ aqueous solutions of the Cdots/[Bmim] $\mathrm{Cl}$ in blue and Cdots/[Bmim] Cl/[Tmi][Trif] in green CdotsCHI/[Bmim]Cl/[Tmi][Trif] in orange, and [Tmi][Trif] in black. (c) Conductivity values obtained at $50{ }^{\circ} \mathrm{C}$ after each heating and cooling cycle of the three pristine nanofluids.

Moreover, these nanofluids present a self-improving feature upon cycling [17,18], which is evident in Figure 3c. Upon four consecutive heating/cooling cycles, the \% increase in the conductivity in Cdots/[Bmim]Cl/[Tmi][Trif] and CdotsCHI/[Bmim]Cl/[Tmi][Trif] nanofluids when compared to Cdots/[Bmim]Cl was 63 and $94 \%$, respectively $[17,18]$. Usually, electrolytes tend to degrade upon consecutive cycling; however, in these nanofluids, we noted precisely the opposite trend (Figure 3b,c). The exact nature of this interesting and quite interesting phenomenon is still not fully understood. However, we believe that the surface traps and holes of the NPs along with the high electron mobility of nanocarbon materials, plus the formation/reformation of hydrogen bonds, provide an innovative pathway for self-improving nanomaterials with increasingly enhanced conductivity. This interesting feature is also observed in 95\% aqueous solutions of the nanofluids (Figure 3b) but not on [Tmi][Trif], thereby demonstrating that this particular property is due to the nanomaterial's inherent nature and chemical composition. Nonetheless, the IL has a good protonic conductivity $\left(0.14 \mathrm{mS} \cdot \mathrm{cm}^{-1}\right.$ at $23^{\circ} \mathrm{C}$ that rises to $0.15 \mathrm{mS} \cdot \mathrm{cm}^{-1}$ at $50{ }^{\circ} \mathrm{C}$ ) and a slight hysteresis behavior that is further boosted in the nanofluids.

A possible problem that may hinder the pristine nanofluids' applications as electrolytes is their high viscosity. Indeed, when considering, for example, the microchannels' batteries technology, their high viscosity could possible lead to a clogging issue. Indeed, the molecular interchange and cohesion forces contribute to the liquid viscosity, but as the temperature increases, the attractive binding energy 
decreases and, consequently, so does the liquid viscosity. This feature can be seen in Figure 4, where the dynamic viscosity decreases progressively with the temperature increase. Cdots/[Bmim] Cl/[Tmi][Trif] nanofluid has a dynamic viscosity of $24 \mathrm{~Pa} \cdot \mathrm{s}$ before performing the first heating cycle at $24{ }^{\circ} \mathrm{C}$, whereas Cdots/[Bmim] Cl has $3.1 \mathrm{mPa} \cdot \mathrm{s}$. Upon the first heating/cooling cycle, there was a drastic drop for the Cdots/[Bmim]Cl viscosity to $1.5 \mathrm{~Pa} \cdot \mathrm{s}$ (94\% decrease) and $2.2 \mathrm{mPa} \cdot \mathrm{s}$ ( $26 \%$ diminution) for Cdots/[Bmim]Cl/[Tmi][Trif] (Figure 3b). Moreover, Figure 3b shows that this trend is maintained over more than five cycles, and even though the sample is left to rest in between cycles for more than $48 \mathrm{~h}$, the dynamic viscosity never reaches the same initial value, which is typical for non-Newtonian flows. In the case of the CdotsCHI/[Bmim] Cl/[Tmi][Trif] nanofluid, there was the formation of crystal-type structures that stopped the dynamic viscosity measurement. These structures are most likely related to particle aggregation [18].
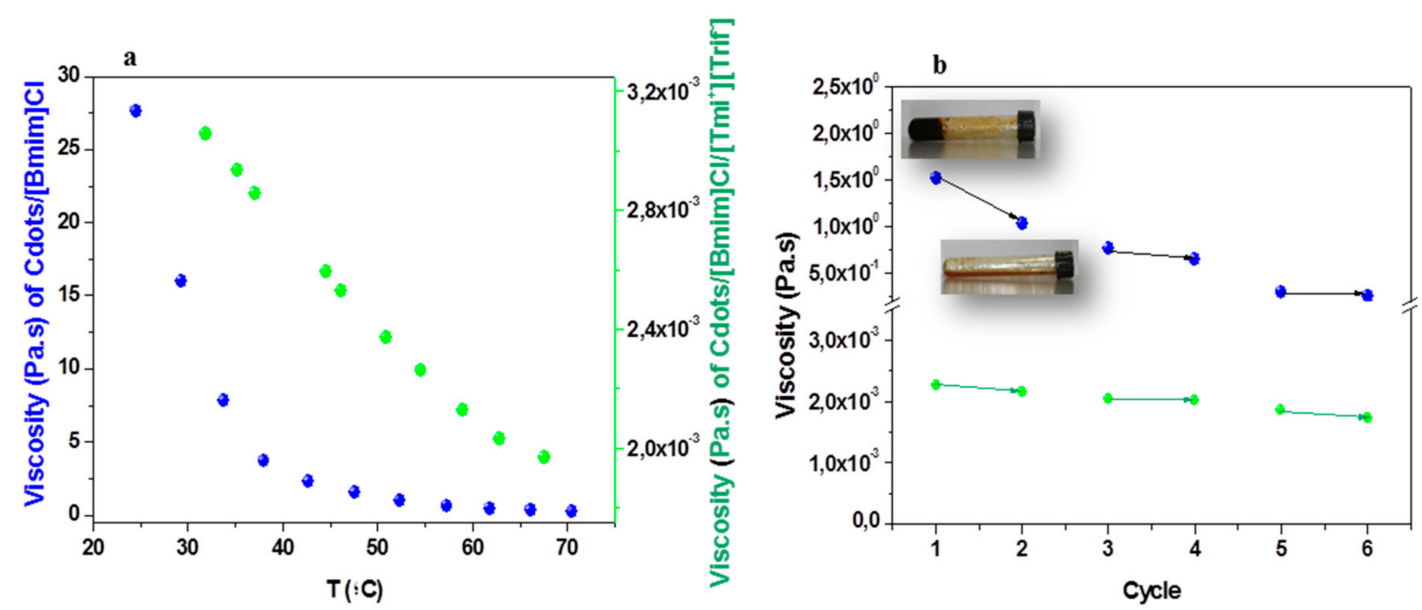

Figure 4. (a) Viscosity profile of the nanofluids in pristine state and (b) viscosity values obtained at $50{ }^{\circ} \mathrm{C}$ after each heating and cooling cycle for $\mathrm{Cdots} /[\mathrm{Bmim}] \mathrm{Cl}$ in blue and $\mathrm{Cdots} /[\mathrm{Bmim}] \mathrm{Cl} /\left[\mathrm{Tmi}^{+}\right]\left[\mathrm{Trif}^{-}\right]$in green. Inset: images of Cdots/[Bmim]Cl before the first heating cycle and upon cooling.

\subsection{Nanofluids Immobilization on PVA Membranes}

The results obtained so far led us to believe that these nanofluids are interesting electrolytes However, we wanted to take these materials to the next level. The use of an electrolyte that can be used in the liquid state, but can also be immobilized in a membrane compatible with these applications could be a major advantage. A major issue in solid-state batteries is the interfaces' stabilization. Indeed, the interfacial composition and structure between solid electrolytes and electrode materials often present major deviations from those of the bulk materials [6]. In a solid-state battery, ion migration is considered a multiscale process. At the atomic scale, mobile cations, such as $\mathrm{Li}^{+} \mathrm{Or} \mathrm{Na}^{+}$, diffuse along favorable migration pathways. These can be seen as ion hops in between ground-state stable sites and/or intermediate metastable sites of the framework constituted by anions, namely, $\mathrm{S}^{2-}$ or polyanionic moieties. Consequently, the availability and interconnectivity of the metastable sites is defined by the anions' arrangements, and this delimits the migration pathway [26], thereby limiting the materials used for immobilization. PVA membranes have been proposed as quite efficient materials for separators in lithium-ion batteries [27,28]. In this sense, we immobilized the nanofluids that exhibited higher conductivities in the nanofluid state, i.e., Cdots/[Bmim] $\mathrm{Cl} /[\mathrm{Tmi}][$ Trif $]$ ) and CdotsCHI/[Bmim]Cl/[Tmi][Trif]) in PVA membranes. For comparison purposes, we also immobilized [Tmi][Trif] in PVA. Several features of the produced PVA membranes were analyzed: morphology, electrolyte uptake/retention, and thermal shrinkage. 


\subsection{Membrane Morphology}

Figure 5 shows the characterization of the PVA-based membranes using atomic force microscopy (AFM). The topographical analysis revealed a medium surface roughness of $471.0 \mathrm{pm}$ for PVA (Figure 5a,b), $0.7067 \mathrm{~nm}$ for PVA incorporating [Tmi][Trif] (Figure 5c,d), $1.886 \mathrm{~nm}$ for PVA with Cdots/[Bmim]Cl/[Tmi][Trif] (Figure 5e,f), and $3.258 \mathrm{~nm}$ for PVA containing CdotsCHI/[Bmim]Cl/[Tmi][Trif] (Figure $5 \mathrm{~g}, \mathrm{~h}$ ). The increase of the surface roughness of PVA upon the introduction of the IL and NPs follows an interesting trend. Indeed, it was noted above $[17,18]$ that CdotsCHI/[Bmim]Cl/[Tmi][Trif] has individual NPs with approximately $44 \mathrm{~nm}$ and larger micelle-like nanometer structures. This size heterogeneity is consistent with the higher medium roughness of the PVA membrane containing this nanofluid.

(a)

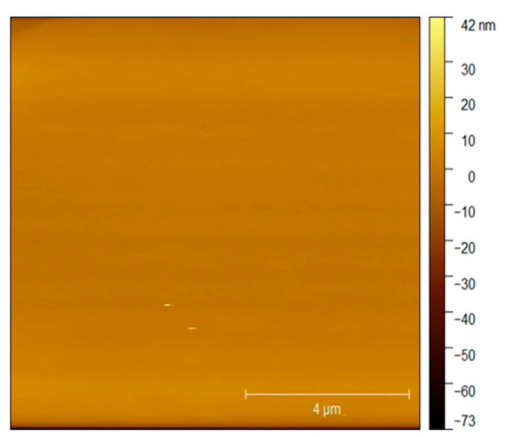

(b)

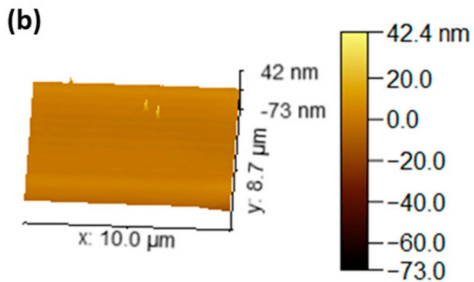

(e)

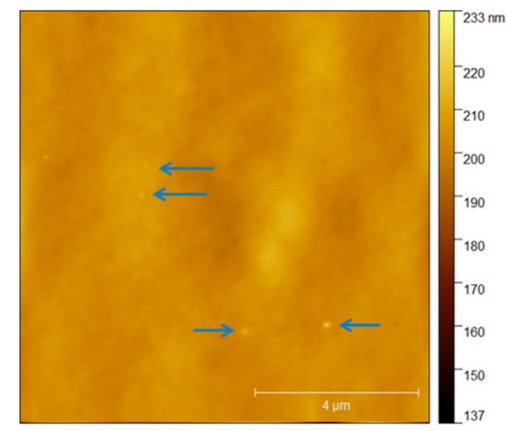

(f)

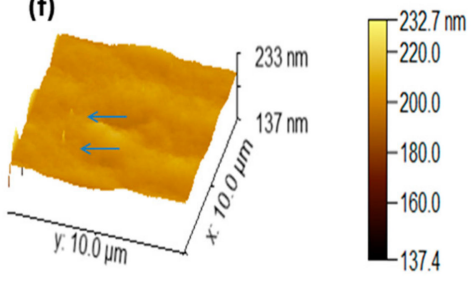

(c)
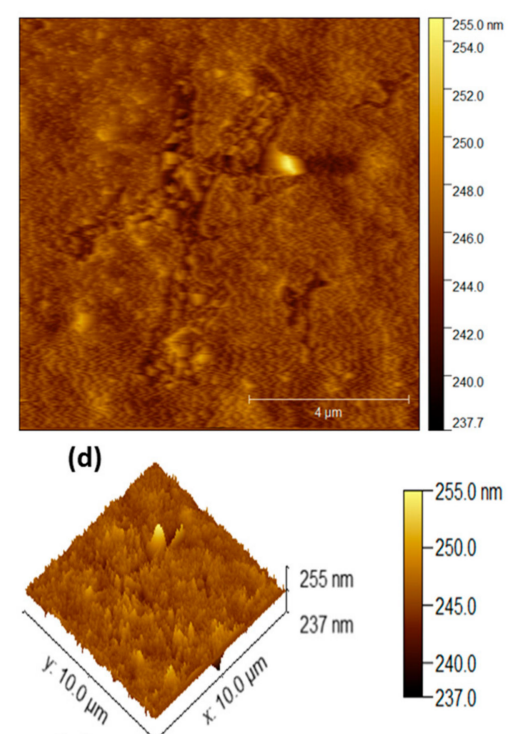

(g)
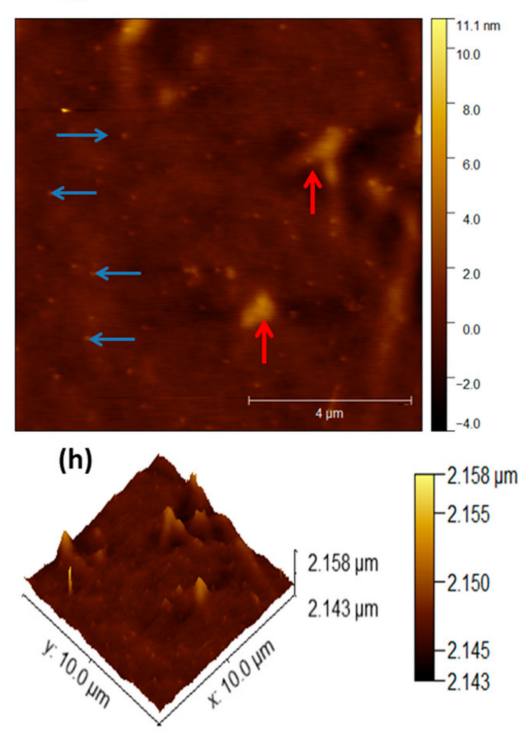

Figure 5. AFM topographical and 3D images of (a,b) PVA membrane; (c,d) PVA membrane with [Tmi][Trif]; (e,f) PVA membrane with Cdots/[Bmim]Cl/[Tmi][Trif]; (g,h) PVA membrane with CdotsCHI/[Bmim]Cl[Tmi][Trif]. 


\subsection{Nanofluids' Immobilization on PVA Membranes}

The membranes were prepared by crosslinking PVA with glutaraldehyde (GA) at $80{ }^{\circ} \mathrm{C}$ upon an activation step provided by $(\mathrm{NCCl})_{3}$. There are several methods for preparing the PVA membranes. Traditionally, chemical crosslinkers, temperature, or physical aging are used (Figure 6). The method used here combined two of the traditional options: A chemical crosslinker (GA) and temperature, $80^{\circ} \mathrm{C}$ (Figure 6). The combination of these two factors allowed us to: (1) Avoid the lack of stability in water that unmodified PVA hydrogels have, which would limit the film performance and integrity [27]; and (2) Increase the crystallinity content provided by the organization of the chains. Indeed, the temperature energy input allowed the establishment of stronger hydrogen bonding among the hydroxyl groups [28].

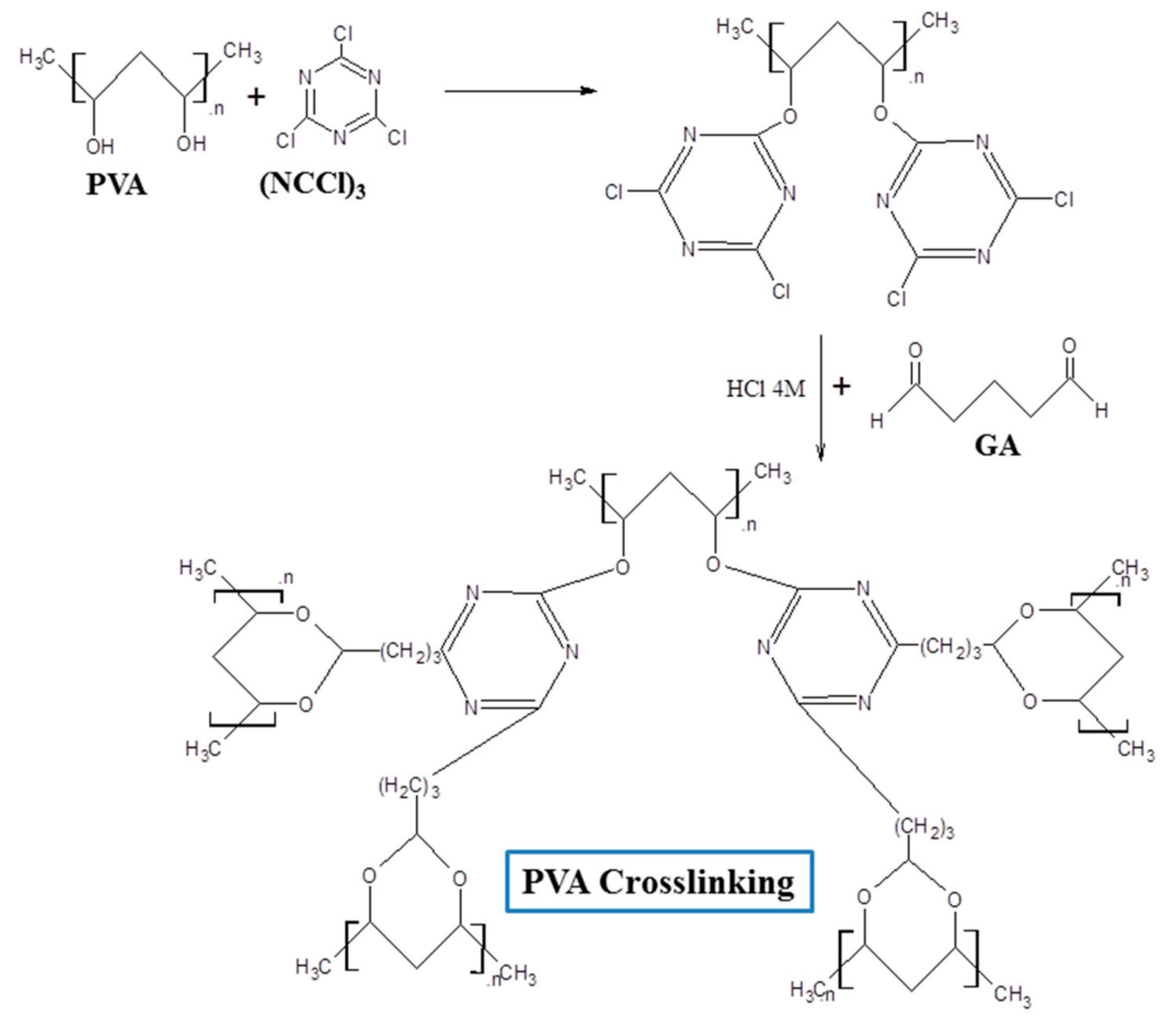

Figure 6. Schematic representation of the PVA membrane formation [29].

The entrapment of Cdots within PVA (Figure 7) was previously accomplished using Cdots with different functionalization, which resulted in refractive index tuning [30], shape-memory effects [31,32], and charge-trapping composites [33]. Indeed, and even though crosslinking of PVA is necessary to increase water resistance, this process has a substantial effect on the optical properties of the entrapped material [34]. 


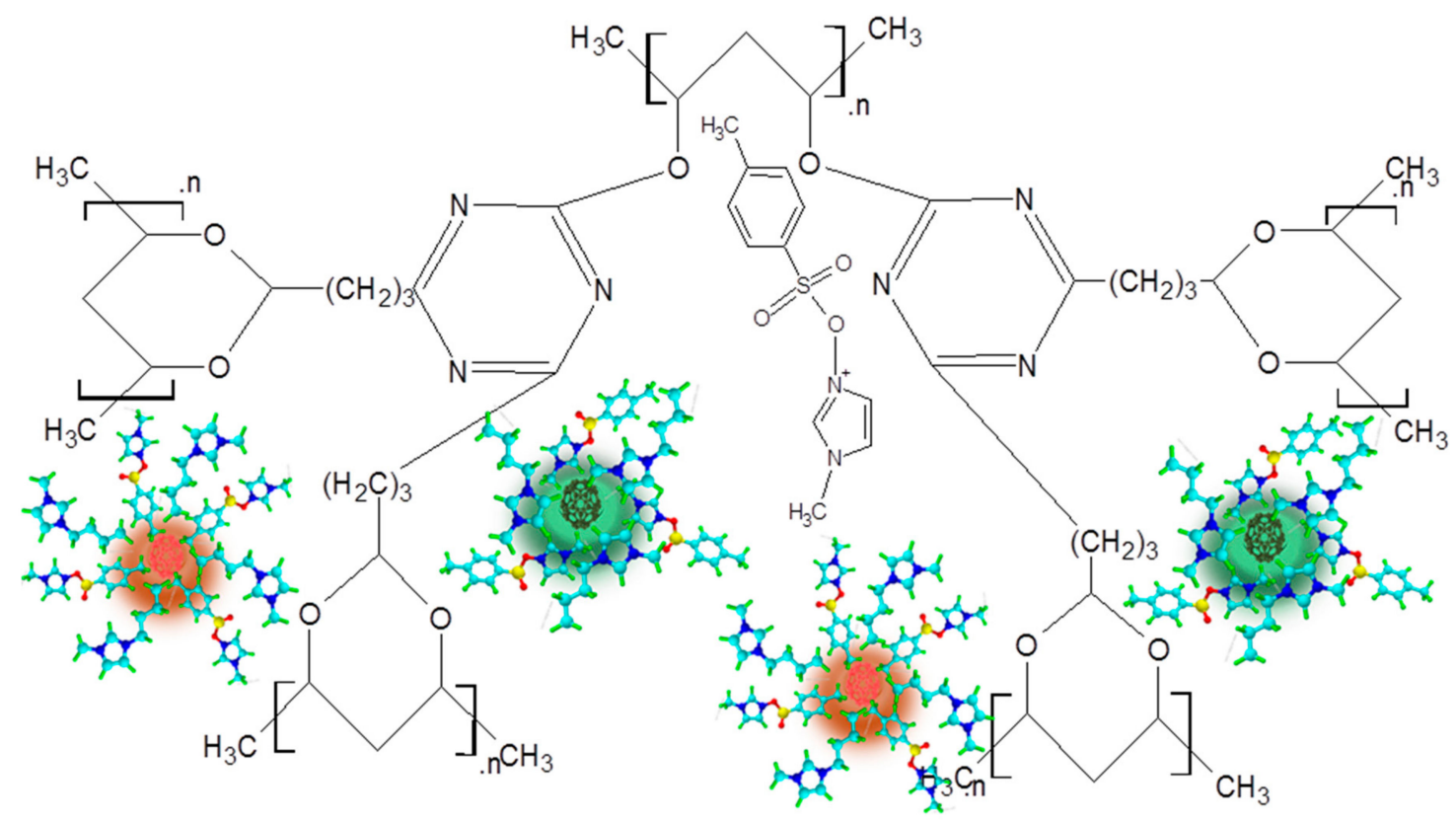

Figure 7. Schematic representation of the entrapment of [Tmi][Trif], Cdots/[Bmim]Cl/[Tmi][Trif], and Cdots/[Bmim]Cl/[Tmi][Trif] in the PVA membrane.

\subsubsection{Study of the Nanofluids' Leaching Profile from the PVA Membranes}

All the PVA membranes had an initial fluorescence profile consistent with the aqueous solution of nanofluids and [Tmi][Trif] (Figure 8). However, we noticed that the fluorescence decreased with time upon membrane production. A systematic study was performed in order to evaluate the membrane fluorescence stability over time.

Figure 8 shows that although the initial fluorescence intensity of all membranes was different, upon $180 \mathrm{~min}$ of production, they all exhibited a similar value. This fluorescence quenching, previously described by Nechifor et al. [34], is due to the chemical interaction between the entrapped species (nanofluids and [Tmi][Trif]) and the crosslinker (GA), which results in an increase in the non-radiative decay mechanism.

Since the water stability was also an important parameter to access, and all the entrapped species were fluorescent, we performed an additional assay where the membranes were immersed in water for $30 \mathrm{~min}$ and left to dry at room temperature for at least 10 consecutive times. The membranes' shape and overall visible stability was conserved during these cycles, pointing out their insolubility in water.

Additionally, we analyzed the fluorescence intensity of the water where the membranes were immersed. It was concluded that the nanofluids and [Tmi][Trif] were successfully entrapped in the PVA membrane and, as such, the quenching effect previously observed was not due to a leaching phenomenon. 

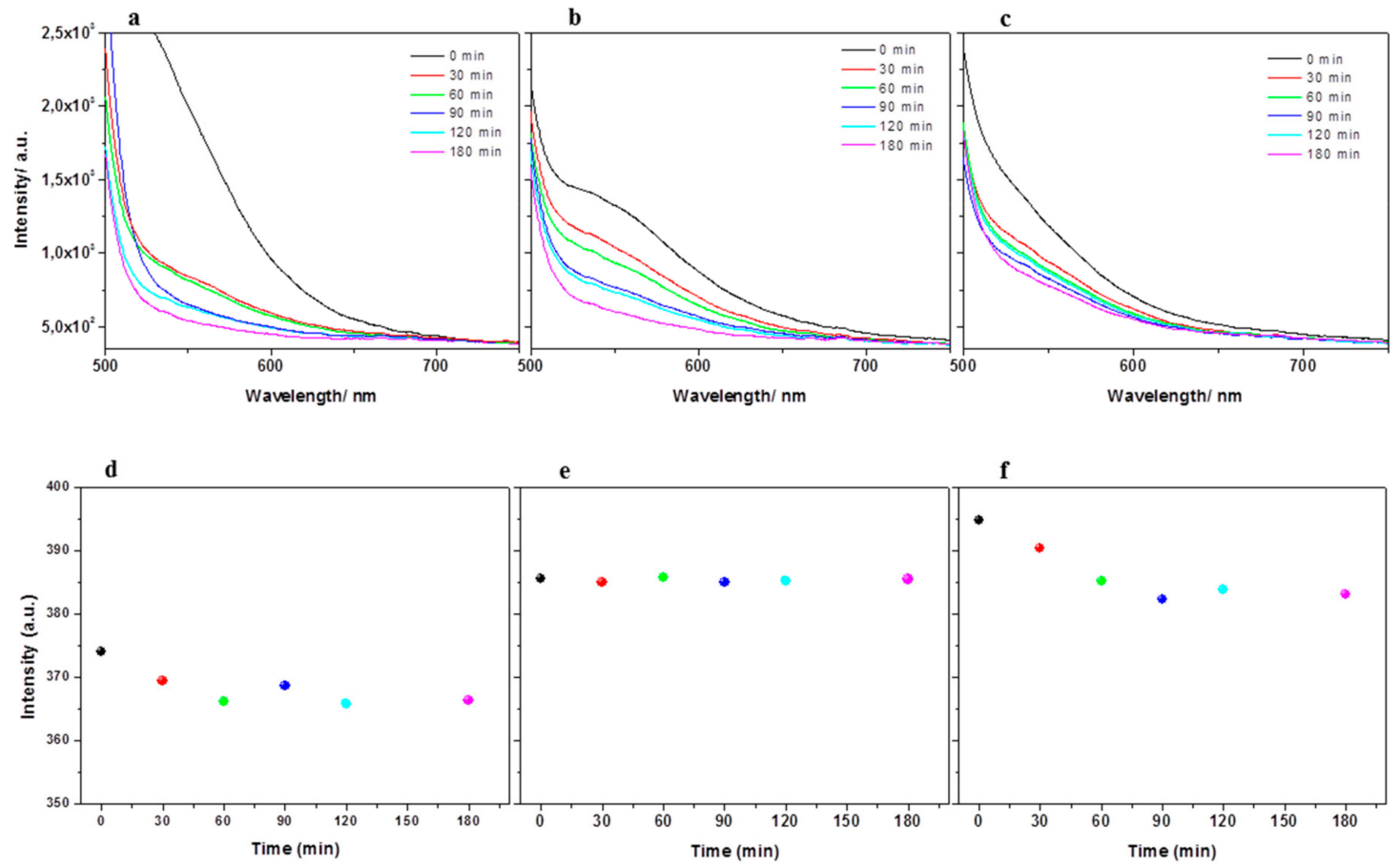

Figure 8. Fluorescence intensity profile in time of (a,d) [Tmi][Trif], (b,e) Cdots/[Bmim]Cl/[Tmi][Trif], and $(\mathbf{c}, \mathbf{f}) \mathrm{CdotsCHI} /[\mathrm{Bmim}] \mathrm{Cl} /[\mathrm{Tm}$ i] $]$ Trif] entrapped in the PVA membranes.

\subsubsection{Degree of Order of the Membranes}

The PVA ability to entrap molecules has been extensively studied [34], and it has been determined that due to the random orientation of the different macromolecular chains, a non-rubbery and non-stretched polymer membrane is usually characterized by an intrinsic optical anisotropy $[35,36]$. Figure 9 shows non-treated visual images of the produced membranes (Figure 9a), the fluorescence image that is similar in all PVA membranes with entrapped species (Figure 9b), and the POM (under crossed polarizers) images of all membranes (Figure 9c-e). Even though PVA has been used in the past in liquid crystal displays [35], non-treated PVA does not show optical birefringence. However, the PVA membranes with entrapped nanofluids and [Tmi][Trif] show birefringence. It has been previously mentioned that the temperature treatment during the PVA crosslinking could lead to an increase in the crystallinity content, and this seems to be the case. Indeed, the presence of thermotropic IL (e.g., $[\mathrm{Bmim}] \mathrm{Cl})$ and their ionic interactions tend to stabilize the lamellar mesophases due to ion-ion stacking and electrostatic interactions [36,37], which leads to birefringence behaviors. This result is quite interesting because a typical feature of IL crystals and semi-crystals is ion conductivity, which can be successfully applied in the development of new anisotropic electric current conductivity devices [37]. 
a

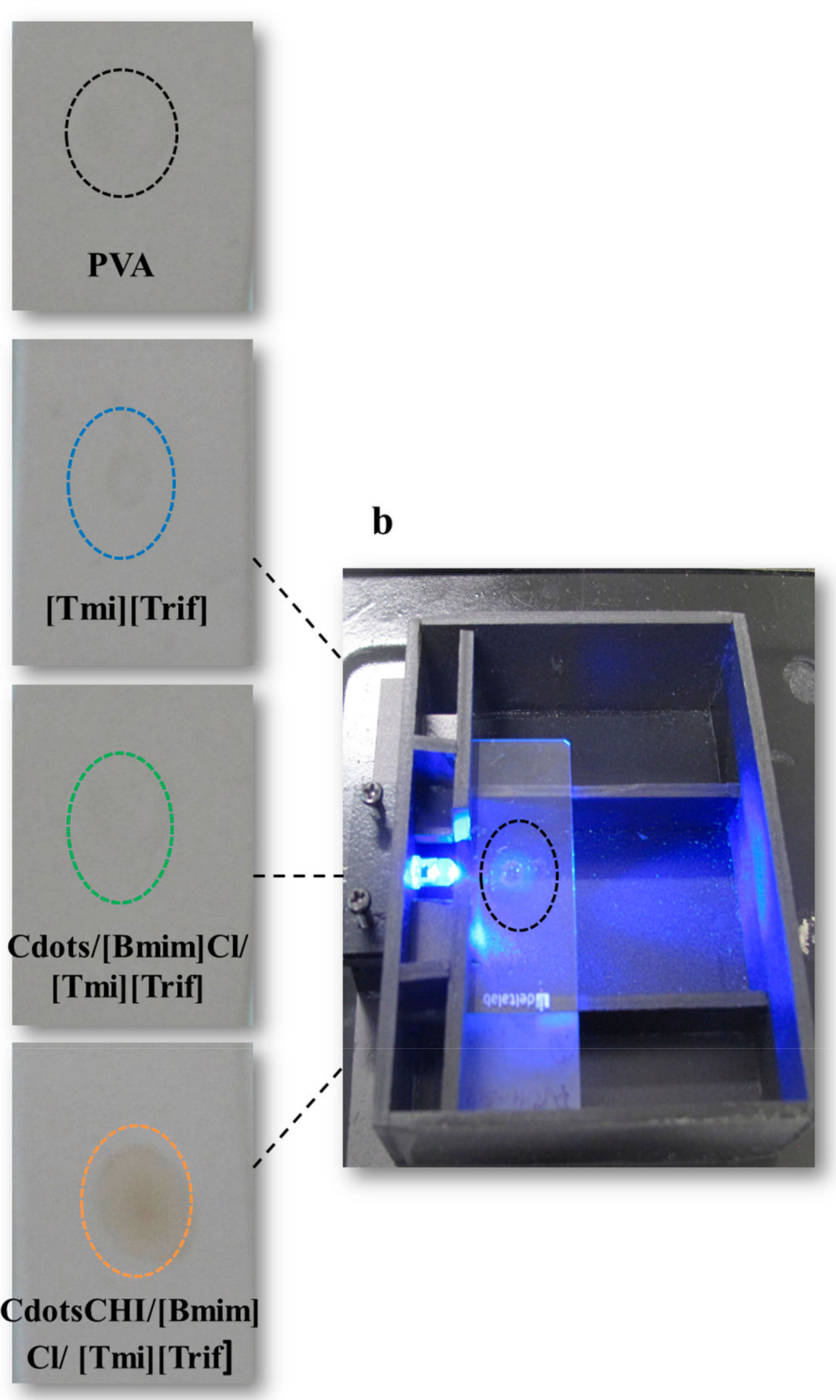

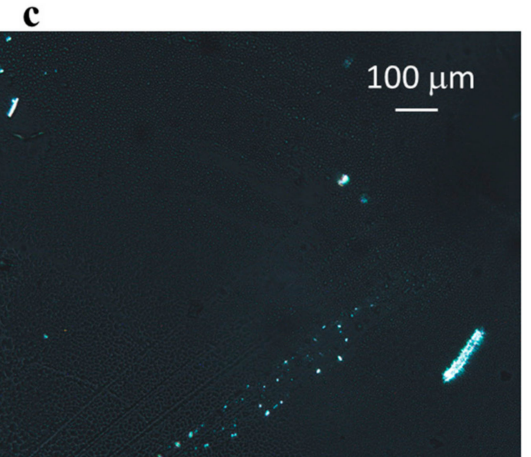
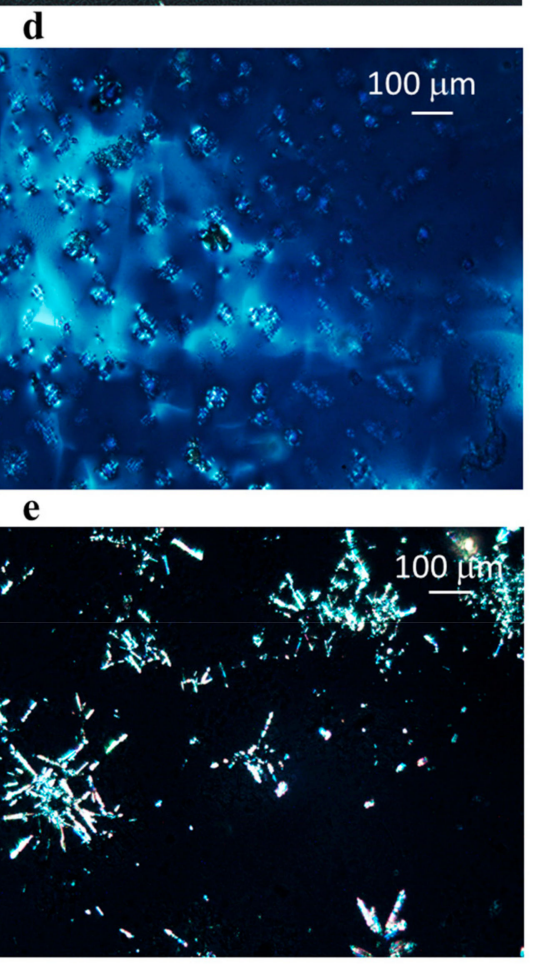

Figure 9. (a) Images of the PVA membranes. (b) Fluorescence image acquired on a homemade device for acquiring the fluorescence of solids using a $450 \mathrm{~nm}$ LED as an excitation source. Polarized optical microscopy images of (c) [Tmi][Trif], (d) Cdots/[Bmim]Cl/[Tmi][Trif], and (e) CdotsCHI/[Bmim]Cl/[Tmi][Trif] entrapped on PVA membranes using a $90^{\circ}$ cross-polarization angle.

\section{Conclusions}

The use of nanofluids in the energy area has been growing due to their particular characteristics. Here, we presented three nanofluids based on Cdots functionalized with two different ILs. The introduction of [Bmim] $\mathrm{Cl}$ and [Tmi][Trif] allowed, not only the formation of the nanofluid, but also defined their protonic conductivity. Indeed, despite the fact that [Tmi][Trif] presents good conductivity, this value is boosted when associated with the intrinsic trap and holes of the NPs. Furthermore, the nanofluids present a self-improving ability characterized by the fact that upon heating/cooling cycles, the conductivity increases and the viscosity decreases, which would be of great benefit in industrial energetic applications. Additionally, these nanofluids were entrapped in a PVA membrane, and despite the fact that their fluorescence intensity was quenched by the interaction of the nanofluids with the crosslinker used, they remained inside the membrane even upon several wetting/drying cycles. Moreover, upon immobilization, and due to the crosslinking process applied to PVA, it was possible to detect birefringent structures. Their visual and morphological characteristics did not change upon wetting/cooling cycles and remained constant for more than four months. Indeed, these nanofluids 
could be an interesting approach to tackle some of the current problems in the fields of solid-state batteries and energy storage, among others.

It must be recalled that it is accepted that our life in the near future will rely on the use of electric vehicles. Their wide use is only hindered by several difficulties the development of advanced rechargeable lithium batteries for energy storage is currently still facing. Properties, such as a high specific surface area, outstanding hierarchical architecture, high thermal and chemical stability, relatively low cost, and benign nature, are some of the most appealing requirements for these energy storage devices. Over the past years, carbon nanotubes (CNTs) and graphene were proposed as two extremely attractive solid candidates [38], with an appealing low cost and noteworthy conductivity. We showed here that the combination of ILs and Cdots is definitely also very interesting, leading not only to an increase in the thermal stability and an enhanced ionic/electronic conductivity, but also to a marked thermosensitivity, which can move the present technology a step forward.

Author Contributions: H.M.R.G. and V.d.Z.B. conceived the project. H.M.R.G., S.A.F.N. and A.D. carried out the experiments. H.M.R.G., S.A.F.N., A.D. and V.d.Z.B. analyzed the experimental data. All authors contributed to writing the manuscript. All authors have read and agreed to the published version of the manuscript.

Funding: This research was funded by National Funds by Foundation for Science and Technology (FCT) and by FEDER funds through POCI-COMPETE 2020-Operational Programme Competitiveness and Internationalization in Axis I - Strengthening research, technological development and innovation (LUMECD (POCI-01-0145-FEDER-016884 and PTDC/CTM-NAN/0956/2014), UniRCell (SAICTPAC/0032/2015 and POCI-01-0145-FEDER-016422), UID/QUI/00616/2013, UID/QUI/50006/2019, PTDC/CTM-NAN/0956/2014, SAICT/PAC/0032/2015, and NORTE-01-0145-FEDER-030858. Helena M.R. Gonçalves and Susana A.F. Neves acknowledge funding by PTDC/BTM-MAT/30858/2017.

Conflicts of Interest: The authors declare no conflict of interest

\section{References}

1. European Commission. "A Roadmap for Moving to a Competitive Low Carbon Economy in 2050", Communication from the Commission to the European Parliament, the Council, the European Economic and Social Committee and the Committee of the Regions, COM (2011) 112. Available online: http: //eur-lex.europa.eu/legal-content/EN/TXT/?uri=CELEX:52011DC0112 (accessed on 10 October 2019).

2. Available online: https://www.nobelprize.org/prizes/chemistry/2019/summary/ (accessed on 10 October 2019).

3. Placke, T.; Kloepsch, R.; Dühnen, S.; Winter, M. Lithium ion, lithium metal, and alternative rechargeable battery technologies: the odyssey for high energy density. J. Solid State Electrochem. 2017, 21, 1939-1964. [CrossRef]

4. Inoue, T.; Mukai, K. Are all-solid-state lithium-ion batteries really safe? Verifcation by diferential scanning calorimetry with an all-inclusive microcell. ACS Appl. Mater. Interfaces 2017, 9, 1507-1515. [CrossRef]

5. Bartsch, T.; Strauss, F.; Hatsukade, T.; Schiele, A.; Kim, A.Y.; Hartmann, P.; Janek, J.; Brezesinski, T. Gas evolution in all-solid-state battery cells. ACS Energy Lett. 2018, 3, 2539-2543. [CrossRef]

6. Famprikis, T.; Canepa, P.; Dawson, J.A.; Islam, M.S.; Masquelier, C. Fundamentals of inorganic solid-state electrolytes for batteries. Nat. Mater. 2019, 18, 1476-4660. [CrossRef] [PubMed]

7. The Future of Batteries Workshop-Preparing a European Initiative on Future Battery Technologies. Available online: https://ec.europa.eu/digital-single-market/en/news/future-batteries-workshop-preparing-europeaninitiative-future-battery-technologies (accessed on 10 October 2019).

8. Dolatabadi, N.; Rahmani, R.; Rahnejat, H.; Garner, C.P. Thermal conductivity and molecular heat transport of nanofluids. RSC Adv. 2019, 9, 2516-5124. [CrossRef]

9. Zheng, Z.; Jing, Q.; Xie, Y.; Zhang, D. An Investigation on the Forced Convection of Al2O3-water Nanofluid Laminar Flow in a Microchannel Under Interval Uncertainties. Appl. Sci. 2019, 9, 432. [CrossRef]

10. Choi, S. Enhancing Thermal Conductivity of Fluids with Nanoparticles. In Developments and Applications of Non-Newtonian Flows; Siginer, D.A., Wang, H.P., Eds.; ASME FED: New York, NY, USA, 1995.

11. Sarkar, J.; Ghosh, P.; Adil, A. A review on hybrid nanofluids: Recent research, development and applications. Renew. Sust. Energ. Rev. 2015, 43, 164-177. [CrossRef]

12. Younes, H.; Ghaferi, A.A.; Saadat, I.; Hong, H.P. Nanofluids Based on Carbon Nanostructures. In Advances in Carbon Nanostructures; Sílva, A., Carabineiro, S., Eds.; BoD-Books on Demand: Norderstedt, Germany, 2016. 
13. Said, Z.; El Haj Assad, M.; Hachicha, A.A.; Bellos, E.; Abdelkareem, M.A.; Alazaizeh, D.Z.; Yousef, B.A.A. Enhancing the performance of automotive radiators using nanofluids. Renew. Sustain. Energy Rev. 2009, 112, 183-194. [CrossRef]

14. MacFarlane, D.R.; Tachikawa, N.; Forsyth, M.; Pringle, J.M.; Howlett, P.C.; Elliott, G.D.; Davis, J.H.; Watanabe, M.; Simon, P.; Angell, C.A. Energy applications of ionic liquids. Energy Environ. Sci. 2014, 7 , 232-250. [CrossRef]

15. Lu, Y.; Korf, K.; Kambe, Y.; Tu, Z.; Archer, L.A. Ionic-Liquid-Nanoparticle Hybrid Electrolytes: Applications in Lithium Metal Batteries. Angew. Chem. Int. Ed. 2013, 53, 488-492. [CrossRef]

16. Minea, A.A.; Sohel Murshed, S.M. A review on development of ionic liquid based nanofluids and their heat transfer behavior. Renew. Sustain. Energy Rev. 2018, 91, 584-599. [CrossRef]

17. Gonçalves, H.M.R.; Pereira, R.F.P.; Lepleux, E.; Carlier, T.; Pacheco, L.; Pereira, S.; Valente, A.J.M.; Fortunato, E.; Duarte, A.J.; de Zea Bermudez, V. Nanofluid Based on Glucose-Derived Carbon Dots Functionalized with [Bmim]Cl for the Next Generation of Smart Windows. Adv. Sustain. Syst. 2019, 3, 1900047. [CrossRef]

18. Gonçalves, H.M.R.; Pereira, R.F.P.; Lepleux, E.; Carlier, T.; Pacheco, L.; Duarte, A.J.; de Zea Bermudez, V. Non-Newtonian Thermosensitive Carbon Dots Nanofluid. 2020, recently submitted.

19. Kim, S.Y.; Kim, S.; Park, M.J. Enhanced proton transport in nanostructured polymer electrolyte/ionic liquid membranes under water-free conditions. Nat. Commun. 2010, 1, 1-7. [CrossRef] [PubMed]

20. Xiao, W.; Zhao, L.; Gong, Y.; Liu, J.; Yan, C. Preparation and performance of poly (vinyl alcohol) porous separator for lithium-ion batteries. J. Membrane Sci. 2015, 487, 221-228. [CrossRef]

21. Havrdova, M.; Hola, K.; Skopalik, J.; Tomankova, K.; Petr, M.; Cepe, K.; Polakova, K.; Tucek, J.; Bourlinos, A.B.; Zboril, R. Toxicity of carbon dots - Effect of surface functionalization on the cell viability, reactive oxygen species generation and cell cycle. Carbon 2016, 99, 238-248. [CrossRef]

22. Briscoe, J.; Marinovic, A.; Sevilla, M.; Dunn, S.; Titirici, M. Biomass-derived carbon quantum dot sensitizers for solid-state nanostructured solar cells. Angew. Chem. Int. Ed. Engl. 2015, 54, 4463-4468. [CrossRef]

23. Sun, X.; Zhang, Q.; Yin, K.; Zhou, S.; Li, H. Fluorescent vesicles formed by simple surfactants induced by oppositely-charged carbon quantum dots. Chem. Commun. 2016, 52, 12024-12027. [CrossRef]

24. Yu, P.; Wen, X.; Toh, Y.-R.; Tang, J. Temperature-Dependent Fluorescence in Carbon Dots. J. Phys. Chem. C. 2012, 116, 25552-25557.

25. Ma, C.; Dai, K.; Hou, H.; Ji, X.; Chen, L.; Ivey, D.G.; We, W. High Ion-Conducting Solid-State Composite Electrolytes with Carbon Quantum Dot Nanofillers. Adv. Sci. 2018, 5, 1700996-1701005. [CrossRef]

26. Figueiredo, K.C.; Alves, T.L.; Borges, C.P. Poly (vinyl alcohol) films crosslinked by glutaraldehyde under mild conditions. J. Appl. Polym. Sci. 2009, 111, 3074-3080. [CrossRef]

27. Hoang, Q.B.; Mai, V.T.; Nguyen, D.K.; Truong, D.Q.; Mai, X.D. Crosslinking induced photoluminescence quenching in polyvinyl alcohol-carbon quantum dot composite. Mater. Today Chem. 2019, 12, 166-172. [CrossRef]

28. Pandey, J.; Qayoom, F.M.; Shukla, A. Synthesis of silica immobilized phosphotungstic acid (Si-PWA)-poly (vinyl alcohol) (PVA) composite ion-exchange membrane for direct methanol fuel cell. Int. J. Hydrog. Energy 2014, 39, 9473-9481. [CrossRef]

29. Kumar, V.B.; Sahu, A.K.; Mohsin, A.S.M.; Li, X.; Gedanken, A. Refractive-index tuning of highly fluorescent carbon dots. ACS Appl. Mater. Interfaces 2017, 9, 28930-28938. [CrossRef] [PubMed]

30. Wu, S.; Li, W.; Zhou, W.; Zhan, Y.; Hu, C.; Zhuang, J.; Zhang, H.; Zhang, X.; Lei, B.; Liu, Y. Large-scale one-step synthesis of carbon dots from yeast extract powder and construction of carbon dots/PVA fluorescent shape memory material. Adv. Opt. Mater. 2018, 6, 1701150. [CrossRef]

31. Yang, G.; Wan, X.; Liu, Y.; Li, R.; Su, Y.; Zeng, X.; Tang, J. Luminescent poly (vinyl alcohol)/ carbon quantum dots composites with tunable water-induced shape memory behavior in different $\mathrm{pH}$ and temperature environments. ACS Appl. Mater. Interfaces 2016, 8, 34744-34754. [CrossRef]

32. Ambasankar, K.N.; Bhattacharjee, L.; Jat, S.K.; Bhattacharjee, R.R.; Mohanta, K. Study of electrical charge storage in polymer-carbon quantum dot composite. Chemistry Select. 2017, 2, 4241-4247. [CrossRef]

33. Cui, Y.; Zola, R.S.; Yang, Y.C.; Yang, D.K. Alignment layers with variable anchoring strengths from polyvinyl alcohol. J. Appl. Phys. 2012, 111, 063520. [CrossRef]

34. Nechifor, C.D.; Postolache, M.; Albu, R.M.; Barzic, A.I.; Dorohoi, D.O. Induced birefringence of rubbed and stretched polyvinyl alcohol foils as alignment layers for nematic molecules. Polym. Adv. Technol. 2019, 30, 2143-2152. [CrossRef] 
35. Nechifor, C.D.; Angheluta, E.; Dorohoi, D.O. Birefringence of etired poly (vinyl alcohol) (PVA) foils. Plast. Mater. 2010, 47, 164-166.

36. Axenov, K.V.; Laschat, S. Thermotropic Ionic Liquid Crystals. Materials 2011, 4, 206-259. [CrossRef] [PubMed]

37. Binnemans, K. Ionic liquid crystals. Chem. Rev. 2005, 105, 4148-4204. [CrossRef] [PubMed]

38. Yuan, W.; Zhang, Y.; Cheng, L.; Wu, H.; Zheng, L.; Zhao, D. The Applications of Carbon Nanotubes and Graphene in Advanced Rechargeable Lithium Batteries. J. Mat. Chem. A 2016,1-20. [CrossRef] 